\title{
Method of characteristics in spherical geometry applied to a Harang-discontinuity situation
}

\author{
O. Amm \\ Technical University of Braunschweig, Institute of Geophysics and Meteorology, Mendelssohnstr. 3, D-38106 Braunschweig, Germany
}

Received: 7 April 1997 / Revised: 28 July 1997 / Accepted: 3 October 1997

\begin{abstract}
The method of characteristics for obtaining spatial distributions of ionospheric electrodynamic parameters from ground-based spatial observations of the ground magnetic disturbance and the ionospheric electric field is presented in spherical geometry. The method includes tools for separation of the external magnetic disturbance, its continuation to the ionosphere, and calculation of ionospheric equivalent currents. Based on these and the measured electric field distribution, the ionospheric Hall conductance is calculated as the primary output of the method. By estimating the Hall- to-Pedersen conductance ratio distribution, the remaining ionospheric electrodynamic parameters are inferred. The method does not assume $\nabla \times \vec{E}=0$ to allow to study time-dependent situations. The application of this method to a Harang discontinuity (HD) situation on 27 October 1977, 17:39 UT, reveals the following: (1) The conductances at and north of the HD are clearly reduced as compared to the eastern electrojet region. (2) Plasma flow across the HD is observed, but almost all horizontal current is diverted into upward-flowing field-aligned currents (FACs) there. (3) The FACs connected to the Hall currents form a latitudinally aligned sheet with a magnitude peak between the electrically and magnetically defined HD, where break-up arcs are often observed. Their magnitude is larger than that of the more uniformly distributed FACs connected to the Pedersen currents. They also cause the southward shift of the magnetically defined HD with respect to the electrically defined one. (4) A tilt of the HD with respect to geomagnetic latitude as proposed by an earlier study on the same event, which used composite vector plot technique, and by statistical studies, is not observed in our single timestep analysis.
\end{abstract}

Also at: Finnish Meteorological Institute, Geophysical Research, P.O. Box 503, FIN-00101 Helsinki, Finland Fax: +49 531391 5220; e-mail: amm@geophys.nat.tu-bs.de
Key words. Ionosphere - Electric fields and currents · Instruments and techniques - Magnetospheric physics . Current systems

\section{Introduction}

During and after the International Magnetospheric Study (IMS, 1976-1979), several methods have been developed to obtain instantaneous spatial distributions of the ionospheric electrodynamic parameters from ground-based measurements of the ground magnetic disturbance field together with the ionospheric electric field. A detailed review of these efforts is given by Untiedt and Baumjohann (1993). The two main approaches have been the "trial and error" or threedimensional modeling method and the method of characteristics.

In the "trial and error" approach (e.g., Baumjohann et al., 1981; Opgenoorth et al., 1983), measurementbased models of the ground magnetic and ionospheric electric field distributions are used together with models of the unknown Hall and Pedersen conductances to vary the latter until the calculated ground magnetic disturbance sufficiently fits the measured one. An advantage of this method is that it can be led through with virtually any data coverage. However, especially (though not only) in case of sparse data, due to the nonunique relation between a certain ground-magnetic field and the ionospheric electrodynamic quantities that produce it, the range of possible models that fit the observations to a specified degree may be quite large, and no error estimation can be provided for the final results. Another disadvantage of the "trial and error" method is that to reproduce the ground magnetic disturbance on a certain model area, the ionospheric quantities have to be modeled on a considerably larger area. Since for that larger area often not enough data of a single time-step are available, it becomes necessary to produce the input 
data by the "composite vector plot" approach which translates temporal changes of the observed quantities into spatial distributions by assuming a stationary structure moving with a certain velocity over the field of view of the measurements (e.g., Kunkel et al., 1986). These assumptions limit the ability of the method to obtain instantaneous output distributions since the stationarity assumption may not hold, and for the uncertainties in the velocity estimation.

The method of characteristics (Inhester et al., 1992; Amm, 1995), on the contrary, is a forward method in which the ionospheric electric and ground magnetic field measurements are used to solve a first-order differential equation for the Hall conductance first. Using the measured electric field and a modeled Hall-to-Pedersen conductance ratio distribution, the remaining electrodynamical quantities are then inferred from it. The typical range of the conductance ratio is relatively narrow, between 1 and 2 for quiet periods (e.g., Schlegel, 1988), increasing up to about 3 under disturbed conditions and about 5 for substorm situations associated with discrete aurora (e.g., Kirkwood et al., 1988; Olsson et al., 1996; Aikio and Kaila, 1996; Lester et al., 1996; however, some extreme values up to 10 directly inside an auroral break-up have been reported by Olsson et al., 1996), and the effect of its modeling on the final results has been shown to be small by Amm (1995). Moreover, additional optical or riometer data may be used for a rough estimate of this ratio. Usually, there are (mostly small) regions where the solution for the Hall conductance is nonunique. However, these regions are known, and an error estimation is available. No data are needed outside the actual area under study. Since divergence and curl of the input data have to be estimated, a reasonable data coverage inside that area is required.

Up to now, both methods have only been used in planar geometry, mostly in the "Kiruna coordinate system" defined by Küppers et al. (1979) which is a stereographic projection of the earth's surface to a tangential plane that touches the earth near Kiruna, Sweden. Whereas neglecting the curvature of earth's surface may be justified for small regions of interest, with the new possibility of obtaining electric field data over nearly half of the northern auroral zone with the SuperDARN radar (Greenwald et al., 1995), the methods should consequently be extended to spherical geometry.

An essential difference between the method of characteristics and the KRM (Kamide et al., 1981), AMIE (Richmond and Kamide, 1988), and IZMEM (Papitashvili et al., 1994) methods that are developed for spherical geometry, too, lies in their different primary input and output quantities: the latter methods use the ground magnetic field (KRM), interplanetary magnetic field (IZMEM), or variable sets of measurements (AMIE) to deduce the ionospheric electric potential, and the conductance distributions are input to them. Since no large-scale spatial conductance measurements are available, these distributions need to a large extent to be taken from statistical models. Whereas this does no harm when studying "typical" or averaged situations, it may restrict the ability of these methods to handle special, instantaneous events. Spatial measurements of the ionospheric electric field as needed as an input to the method of characteristics are available from coherent-scatter radars. However, the application of the method is limited to situations in which the radars receive enough backscatter to provide electric field data on a sufficiently large area.

In this paper, we will derive the method of characteristics for spherical geometry, including tools for extraction of the external part of the ground magnetic disturbance, its continuation to the ionosphere and calculation of the ionospheric equivalent currents.

We will then apply the method, using magnetic field data from the Scandinavian Magnetometer Array (SMA) (Küppers et al., 1979) and electric field data from the STARE coherent-scatter radar (Nielsen, 1982), on a Harang-discontinuity situation on 27 October 1977. We have selected this event for three different reasons: (1) Densely spaced ground magnetic field data from the SMA and STARE electric field data with good backscatter directly over that array are available. (2) This event was studied earlier by Lampen (1985) [main results shown in Untiedt and Baumjohann (1993)], so that we can compare our results using the method of characteristics with those gained by the "trial and error" method. (3) Finally, with the findings of this study we try to address some questions raised by Koskinen and Pulkkinen (1995) in their review on the physics of the Harang discontinuity.

\section{Theory}

\subsection{General}

The method of characteristics requires as input distributions of the ground magnetic field disturbance $\vec{B}_{G}$, the ionospheric electric field $\vec{E}$, and the ratio of the Hall to Pedersen conductance $\alpha$. The first task is to extract the external part of $\vec{B}_{G}$, then continue it to the ionospheric height, and therefrom derive the ionospheric equivalent currents $\vec{J}_{\text {eq,Ion }}$ (Sect. 2.2). The ionosphere is treated as an infinitely thin conducting layer at radius $R_{I}=R_{E}+100 \mathrm{~km}$, with $R_{E}$ being the earth's radius. Next, we have to prove that $\vec{J}_{\text {eq,Ion }}$ is equal to the divergence-free part of the real horizontal ionospheric sheet currents, $\vec{J}_{d f}$ (Sect. 2.3). Then, the "core equations" of the method are discussed in Sect. 2.4. Finally, in Sect. 2.5, we take a closer look at time-dependent situations.

As in previous studies (e.g., Fukushima, 1976; Untiedt and Baumjohann, 1993), we assume that the earth's main magnetic field is directed straight and perpendicular to the ionosphere. Moreover, we assume that there is no deviation between the magnetic field lines and the flow direction of the field-aligned currents $j_{\|}$(FACs). Consequently, in spherical geometry the FACs flow radially. Deviations of the real FAC flow from that direction are in our context only important 
because of the ground magnetic field that this deviation causes (see Sect. 2.3), which has been shown to be small in polar latitudes (cf. Richmond, 1974; Fukushima, 1976; Tamao, 1986; Untiedt and Baumjohann, 1993, their Fig. 12; Amm, 1995). However, in mid- and especially equatorial latitudes the effect of that deviation becomes important, so that there we cannot use the method described in this paper (see also Sect. 2.4). A detailed discussion on the ground magnetic effect of straight but tilted FACs in planar geometry, including analytical expressions, and on the possible, but small errors in the output quantities of the method of characteristics related to that can be found in Amm (1995).

2.2 Separation of the external magnetic disturbance field, continuation to the ionosphere and calculation of ionospheric equivalent currents: spherical cap harmonic analysis

Decomposition of the magnetic field disturbance $\vec{B}_{G}$ into its parts due to internal and external sources, and its height continuation by means of a spherical harmonic analysis (SHA) go back to Gauß and is fully elaborated as early as in the classical book of Chapman and Bartels (1940). However, since the basis functions of SHA extend over the whole earth, problems appear if the area of interest and of measurements is confined to a part of the earth's surface only: the SHA coefficients will then be poorly defined, or "virtual" data points have to be added. Moreover, since the maximum wavelength resolution of an SHA analysis is $\lambda_{\min }=\left(2 \pi R_{E}\right) / n_{\max }$ where $n_{\max }$ is the maximum degree used in the analysis, for a desired resolution of $\lambda_{\min }=400 \mathrm{~km}$, $n_{\max }=100$, i.e., a total number of 10201 coefficients would be required.

A way out of these problems is provided by spherical cap harmonic analysis (SCHA) (Haines, 1985). Let us assume that a given data set can be covered by a spherical cap with midpoint $\left(\vartheta_{p}, \varphi_{p}\right)$ (in geographical coordinates) and a half-angle $\vartheta_{0}$ of the cap. The SCHA expansion of the magnetic potential $\Phi$ in the current-free region between $r=R_{E}$ and $r=R_{I}$ in the spherical coordinate system with the midpoint of the cap as the northern pole is (Haines, 1985)

$$
\begin{aligned}
\Phi(r, \vartheta, \varphi)= & R_{E}\left[\sum_{k=0}^{K_{i}} \sum_{m=0}^{k}\left(\frac{R_{E}}{r}\right)^{n_{k}(m)+1} P_{n_{k}(m)}^{m}(\cos \vartheta)\right. \\
& \cdot\left\{g_{k}^{m, i} \cos (m \varphi)+h_{k}^{m, i} \sin (m \varphi)\right\} \\
& +\sum_{k=0}^{K_{e}} \sum_{m=0}^{k}\left(\frac{r}{R_{E}}\right)^{n_{k}(m)} P_{n_{k}(m)}^{m}(\cos \vartheta) \\
& \left.\cdot\left\{g_{k}^{m, e} \cos (m \varphi)+h_{k}^{m, e} \sin (m \varphi)\right\}\right]
\end{aligned}
$$

The first term of this equation gives the internal, the second sum the external part of the magnetic potential. Both of them can independently be calculated at different radii by inserting respective values for $r$. The structure of Eq. (1) is similar to the corresponding one of SHA, but to yield appropriate basis functions on the cap, the SHA integral degree $n$ has to be replaced by a SCHA nonintegral degree $n_{k}(m)$ where $k=0, \ldots, K_{i}$ for the sum of internal coefficients (index ' $i$ ') and $k=0, \ldots, K_{e}$ for external coefficients (index ' $e$ ') is an integer, with $K_{i}$ and $K_{e}$ determining the number of internal and external coefficients to be taken into account for the expansion of the potential. The $n_{k}(m)$ are determined by the boundary conditions for the associated Legendre functions $P_{n_{k}(m)}^{m}(\cos \vartheta)$ at $\vartheta=\vartheta_{0}$

$$
\begin{aligned}
\frac{\mathrm{d} P_{n_{k}(m)}^{m}\left(\cos \vartheta_{0}\right)}{\mathrm{d} \vartheta}=0 & \text { for } k-m \text { even, } \\
P_{n_{k}(m)}^{m}\left(\cos \vartheta_{0}\right)=0 & \text { for } k-m \text { odd }
\end{aligned}
$$

i.e., for a given $m$ and $\vartheta_{0}$, those Legendre functions which fulfil Eq. (2) are searched with increasing $n_{k}(m)$ and are indexed by $k$. Accordingly, a definition of Legendre functions is needed that does not rely on an integer degree $n$ (Hobson, 1931; Haines, 1985):

$$
\begin{aligned}
P_{n}^{m}(\cos \vartheta)= & K_{n}^{m} \cdot \sin ^{m} \vartheta \\
& \cdot F\left(m-n ; n+m+1 ; 1+m ; \frac{1-\cos \vartheta}{2}\right),
\end{aligned}
$$

\begin{tabular}{|c|c|c|c|c|c|c|c|c|c|}
\hline$\downarrow k m \rightarrow$ & 0 & 1 & 2 & 3 & 4 & 5 & 6 & 7 & 8 \\
\hline 0 & 0.00 & & & & & & & & \\
\hline 1 & 6.38 & 4.84 & & & & & & & \\
\hline 2 & 10.49 & 10.49 & 8.36 & & & & & & \\
\hline 3 & 15.31 & 14.79 & 14.26 & 11.69 & & & & & \\
\hline 4 & 19.60 & 19.60 & 18.75 & 17.86 & 14.93 & & & & \\
\hline 5 & 24.29 & 23.97 & 23.64 & 22.53 & 21.36 & 18.13 & & & \\
\hline 6 & 28.65 & 28.65 & 28.09 & 27.52 & 26.20 & 24.79 & 21.29 & & \\
\hline 7 & 33.28 & 33.04 & 32.81 & 32.05 & 31.28 & 29.78 & 28.18 & 24.43 & \\
\hline 8 & 37.67 & 37.67 & 37.25 & 36.83 & 35.91 & 34.97 & 33.30 & 31.52 & 27.55 \\
\hline
\end{tabular}

where $F(\alpha ; \beta ; \gamma ; x)$ is the hypergeometric function and $K_{n}^{m}$ are normalization factors [in geophysics usually Schmidt normalization, e.g., Chapman and Bartels (1940)].

One important fact to note is that $n_{k}(m) \geq k$ (see Table 1 for an example with $\vartheta_{0}=20^{\circ}$ and $\left.k=0, \ldots, 8\right)$.

Table 1. Spherical cap noninteger degrees $n_{k}(m)$ for cap half angle $\vartheta_{0}=20^{\circ}$ 
The minimum wavelength resolution of SCHA is similar to that already given for SHA, but with $n_{\max }$ substituted by $n_{k}(m)_{\max }$. For the example as given in Table 1, an SHA analysis with the same number of coefficients would have $n_{\max }=8$. As seen from the table, for SCHA $n_{k}(m)_{\max }=37.67$ holds. Hence, for our example we see that with the same number of coefficients, SCHA yields an about 4.7 times higher spatial resolution than SHA, in addition to the already-stated advantage that no unsampled data outside the spherical cap is needed.

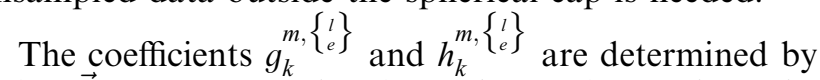
fitting $\vec{B}_{G}=-\nabla \Phi$ to the given observations. Then, the external part of the magnetic disturbance is extracted and the height continuation is performed by taking only the second sum and setting $r=R_{I}$ in Eq. (1). Computer programs for these purposes were given by Haines (1988).

Two notes of caution should be given: (1) As noted by Haines (1985), errors can occur at the boundary $\vartheta=\vartheta_{0}$ during the upward continuation and spread inward with increasing continuation height. Haines attributed these errors to the missing independent control of the second and higher derivatives at that boundary. From our experience with the continuation from the earth's surface to the ionosphere, it is enough to select $\vartheta_{0}$ 1-2 $2^{\circ}$ larger than demanded by the data points or the region of interest to prevent this effect from influencing that region. (2) Torta and de Santis (1996) found that SCHA may encounter numerical problems in the process of field separation when long wavelengths are modeled over small caps (similar to problems that occur in Fourier transforms when wavelengths longer than the transformation area are present). Their and our own experiences agree in that these errors can become critical for the small and often quite homogeneous internal part, but are not remarkable for the larger, usually more structured external part which is solely of interest for this study.

In planar geometry, the relation between the magnetic field disturbance immediately below or above a plane and the related equivalent currents in that plane is local $\left(\vec{J}_{\text {eq }}(\vec{r})=2 / \mu_{0}(\hat{z} \times \vec{B}(\vec{r}))\right.$, where $\hat{z}$ is a unit vector perpendicular to the plane and $\vec{B}$ the magnetic field in the immediate vicinity of the plane, if it is left in $\hat{z}$ direction) since any segment of $\vec{J}_{e q}$ causes a horizontal magnetic disturbance directly below and above its position only, and elsewhere in the plane a purely vertical one. In spherical geometry, this relation becomes nonlocal. Expressed in spherical (cap) harmonics, where $\Phi_{k}^{e}$ denotes the $k$-th harmonic of the external magnetic potential [i.e., the external part of Eq. (1) inside the $k$ sum] immediately below the ionosphere, and $\Psi_{k}$ the corresponding $k$-th harmonic of the ionospheric equivalent current function as defined by $\vec{J}_{e q, I o n}=+\nabla \Psi$, we get [cf. Richmond, 1974; Haines and Torta, 1994; the factor $10 / 4 \pi$ that appears in Chapman and Bartels (1940) and Kamide et al. (1976) is related to cgs units]

$\Psi_{k}=\frac{1}{\mu_{0}}\left(2-\frac{1}{n-1}\right) \Phi_{k}^{e}$.
Note that Eq. (4) can be inserted into Eq. (1) to calculate $\Psi$ from the SCHA coefficients in a one-step procedure. The constant factor $2 / \mu_{0}$ is the same as in planar geometry and represents the local part of the relation between $\Psi$ and $\Phi^{e}$, whereas the second term in brackets is responsible for the nonlocal part. The latter disappears for $n \rightarrow \infty$, corresponding to $\lambda \rightarrow 0$ when the curvature of the sphere becomes negligible. To show this nonlocality, Fig. 1 displays the horizontal magnetic field of a constant sheet current of $1000 \mathrm{~mA} / \mathrm{m}$, flowing eastward with its center at constant latitude of $70^{\circ}$ (the sheet current has $1^{\circ}$ of width and extends over $180^{\circ}$ longitude), as calculated by direct Biot-Savart integration. To make the small vectors due to the nonlocal part of Eq. (4) visible, we deleted the 637-nT vectors produced by the local part at the center latitude of the current flow. In the case shown, the nonlocal vectors have a magnitude of about $0.6 \%$ of the local ones at $1^{\circ}$ latitudinal distance of the current, and decrease to $0.18 \%$ at $5^{\circ}$ latitudinal distance.

In some studies, the three steps presented in this section (i.e., extraction of the external part of the magnetic potential, its continuation to the ionosphere, and calculation of equivalent currents considering spherical geometry) are completely skipped (e.g., Lühr and Schlegel, 1994; Stauning, 1995), and the ionospheric equivalent currents are approximated as $\vec{J}_{e q, I o n} \approx 2 / \mu_{0}$ $\left(\vec{B}_{G} \times \hat{r}\right)$, i.e., as equal to the total equivalent currents on the ground. However, we feel that although depending on the type and purpose of the study, considerable errors can be introduced, especially if the upward continuation is skipped. Typically, the ionospheric equivalent currents are not only larger than those on the ground, but may also have a somewhat different structure, since high wave numbers are amplified in the process of upward continuation [cf. Eq. (1), compare Sect. 3].

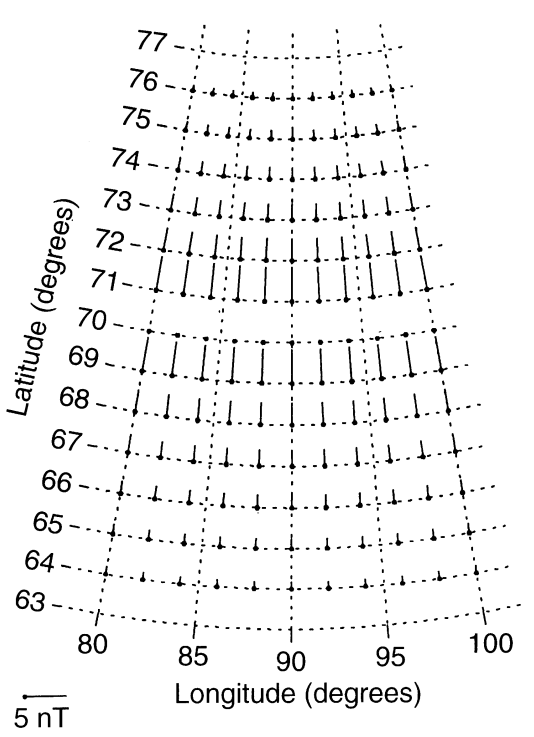

Fig. 1. Magnetic field of an eastward-flowing ionospheric sheet current of $1000 \mathrm{~mA} / \mathrm{m}$ at $70^{\circ}$ latitude, immediately below the ionosphere; the 637-nT magnetic field vectors at the latitude of current flow have been deleted to show the small "nonlocal" effect between current and horizontal magnetic field on the sphere 
2.3 Ionospheric equivalent currents and divergence-free part of real ionospheric currents

As with any vector field, we can decompose the real ionospheric sheet current density $\vec{J}$ into a curl-free and a divergence-free part uniquely:

$\vec{J}=\vec{J}_{c f}+\vec{J}_{d f}$.

For $\vec{J}_{e q, I o n}$ to be equal to $\vec{J}_{d f}$ it is thus required that $\vec{J}_{c f}$ together with its accompanying FACs

$j_{\|}=\nabla_{h} \cdot \vec{J}=\nabla_{h} \cdot \vec{J}_{c f}$

causes no magnetic field below the ionosphere (the subscript $h$ denotes the horizontal part of the divergence vector operator). This can be most easily proved by decomposing $\vec{J}$ into spherical curl-free and divergencefree elementary current system (Amm, 1997; note that this can be done regardless of any considerations on conductances or the electric field), and then proving that the curl-free elementary system together with its FACs has the desired property. The curl-free elementary system is

$\vec{J}_{c f, e l}=\frac{I_{0, c f}}{4 \pi R_{I}} \cot \left(\vartheta^{\prime} / 2\right) \underline{e}_{\vartheta^{\prime}}$,

where $\vartheta^{\prime}=0$ is called the pole of the elementary system. Equation (7) is similar to a system that was attributed to Pedersen currents in an earlier study by Fukushima (1976). There and in Amm (1997) proofs are given that the magnetic effect of the this system together with its radially flowing FACs is zero below the ionosphere. Hence, we have

$\vec{J}_{e q, I o n}=\vec{J}_{d f}$

\subsection{Core equations of the method of characteristics}

To summarize shortly our start equation, we have $\vec{J}_{\text {eq,Ion }}=$ Fct. $\left(\vec{B}_{G}\right)$ from Sect. 2.2, and Eq. (5) together with Eq. (8) as the link between $\vec{J}_{e q, I o n}$ and $\vec{J}$ from Sect. 2.3. Also noted in that section is Eq. (6) as the relation between $\vec{J}$ and its FACs that comes from the divergence-freeness of the total current system. In its given form, it is valid for the earth's main magnetic field being perpendicular to the ionosphere, as assumed. Finally, we need Ohm's law which, under the same assumption, has the form

$$
\left(\begin{array}{l}
J_{\vartheta} \\
J_{\varphi}
\end{array}\right)=\left(\begin{array}{cc}
\Sigma_{P} & -\Sigma_{H} \\
\Sigma_{H} & \Sigma_{P}
\end{array}\right) \cdot\left(\begin{array}{c}
E_{\vartheta} \\
E_{\varphi}
\end{array}\right) .
$$

However, in the real case the preceding assumption is not fulfilled and the tensor $\Sigma$ in Eq. (9), including polarization effects, should rea (e.g., Amm, 1996)

$\underline{\underline{\Sigma}}=\left(\begin{array}{cc}\frac{\Sigma_{0} \Sigma_{P}}{C} & \frac{\Sigma_{0} \Sigma_{H}(-\cos \varepsilon)}{C^{C}} \\ \frac{\Sigma_{0} \Sigma_{H} \cos \varepsilon}{C} & \Sigma_{P}+\frac{\Sigma_{H}^{2} \sin ^{2} \varepsilon}{C}\end{array}\right)$,

where $\Sigma_{0}$ is the conductance parallel to the magnetic field, $\varepsilon$ the angle between the magnetic field lines and the normal on the ionosphere, and $C=\Sigma_{0} \cos ^{2} \varepsilon+\Sigma_{P} \sin ^{2} \varepsilon$. The question arises, what errors are introduced for $\underline{\underline{\Sigma}}$ by the assumption of a radial main magnetic field. Figure 2 shows the tensor elements of Eq. (10) for a dipole main magnetic field, with $\Sigma_{P} \equiv 1$ and $\Sigma_{H} \equiv 2$ (in relative units) which are also indicated in Fig. 2 by horizontal dotted lines. The deviation between the tensor elements of Eqs. (9) and (10) is nearly unnoticeable for $\vartheta \leq 30^{\circ}$, i.e., polar latitudes, still acceptable for $\vartheta \leq 45^{\circ}$, but then increases dramatically toward the equator. Thus, in addition to the considerations of Sect. 2.1, this is another reason why the method presented cannot be used in low latitudes.

After the start equations are verified, the remaining considerations are completely similar to those for planar geometry given by Inhester et al. (1992) and Amm (1995). This is because the remaining "core equations" of the method of characteristics are local and can thus be used in any geometry as long as the start equations hold (of course, the differential operators must be expanded according to the respective geometry). For completeness, we give a short summary of those equations and refer to the already cited for more detail.

We define a vector field

$\vec{V}=\vec{E}-\alpha^{-1} \vec{E} \times \underline{e}_{r}$

and two scalar fields

$C=\nabla_{h} \cdot \vec{V}$,

$D=-\left[\nabla \times \vec{J}_{e q, I o n}\right]_{r}$.

By combining Eqs. (9) and (8) we obtain

$\frac{1}{R_{I}} \frac{\partial \Sigma_{H}}{\partial \vartheta} V_{\vartheta}+\frac{1}{R_{I} \sin \vartheta} \frac{\partial \Sigma_{H}}{\partial \varphi} V_{\varphi}=D-C \Sigma_{H}$.

This first-order partial differential equation can be split up into many first-order ordinary differential equations

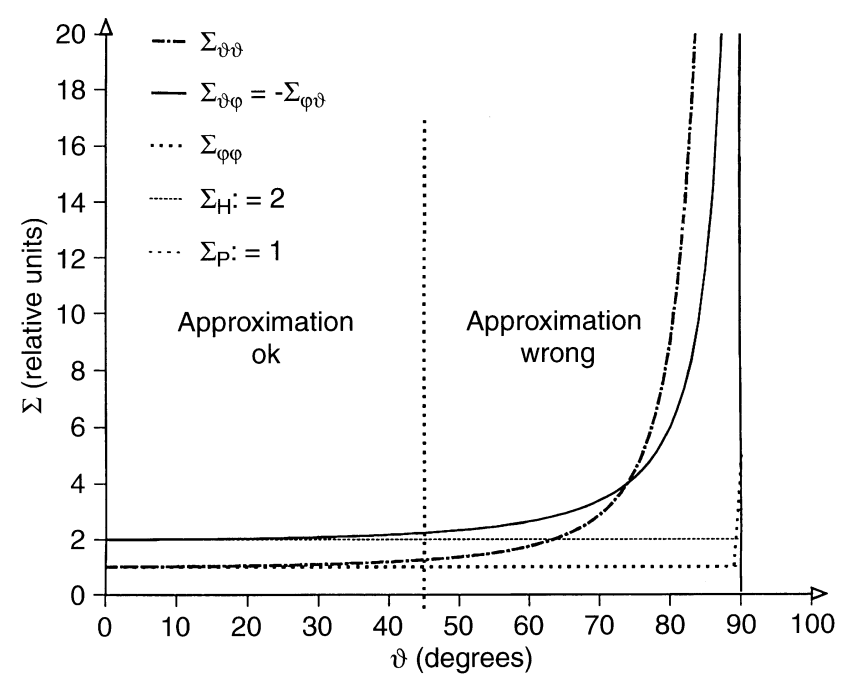

Fig. 2. Components of conductance tensor $\Sigma$, including polarization effects, for a dipole main magnetic field; in rēative units, with $\Sigma_{H} \equiv 2$ and $\Sigma_{P} \equiv 1$ (see horizontal dotted lines) 


$$
\frac{\mathrm{d} \Sigma_{H}(\vec{r}(\ell))}{\mathrm{d} \ell}+\frac{C(\vec{r}(\ell))}{|\vec{V}(\vec{r}(\ell))|} \cdot \Sigma_{H}(\vec{r}(\ell))=\frac{D(\vec{r}(\ell))}{|\vec{V}(\vec{r}(\ell))|}
$$

where $\vec{r}(\ell)$ are the characteristics of Eq. (14) with the geometric path length $\ell$ defined by

$\frac{\mathrm{d}}{\mathrm{d} \ell} \vec{r}(\ell)=\frac{1}{|\vec{V}(\vec{r}(\ell))|}\left(\begin{array}{c}V_{\vartheta}(\vec{r}(\ell)) / R_{I} \\ V_{\varphi}(\vec{r}(\ell)) / R_{I} \sin \varphi\end{array}\right)$,

i.e., they are everywhere tangential to $\vec{V}$. Equation (15) can be solved straightforwardly to

$\Sigma_{H}(\vec{r}(\ell))=\Sigma_{H}\left(\vec{r}_{0}\right) \mathrm{e}^{-I(0, \ell)}+\int_{0}^{\ell} \frac{D\left(\vec{r}\left(\ell^{\prime}\right)\right)}{\mid \vec{V}\left(\vec{r}\left(\ell^{\prime}\right) \mid\right.} \mathrm{e}^{-I\left(\ell^{\prime}, \ell\right)} \mathrm{d} \ell^{\prime}$

with

$I\left(\ell^{\prime}, \ell\right)=\int_{\ell^{\prime}}^{\ell} \frac{C\left(\vec{r}\left(\ell^{\prime \prime}\right)\right)}{\left|\vec{V}\left(\vec{r}\left(\ell^{\prime \prime}\right)\right)\right|} \mathrm{d} \ell^{\prime \prime}$

The solution provided by Eq. (17) consists of a term dependent on the unknown initial value $\Sigma_{H}\left(\vec{r}_{0}\right)$ (often, but not necessarily, given on a boundary point of the region of interest) and another part solely determined by the input quantities. The influence of $\Sigma_{H}\left(\vec{r}_{0}\right)$, however, will decrease exponentially with (positive) $I(0, \ell)$ and will therefore usually be marginal for most part of a characteristic. If $I(0, \ell)$ is mainly negative along a characteristic, the direction of integration is changed from $+\vec{V}$ to $-\vec{V}$ direction, causing the sign of $I$ to switch, too. Since characteristics intersect the boundary of the region under study twice (except for when they reach a "singular point", see below), and the direction of integration is chosen as already described such that the characteristics spread up in that direction, boundary values have to be given on at most $50 \%$ of the boundary, often much less (see Sect. 3). That part of the boundary is called "influencing".

An error estimation for regions where the solution of $\Sigma_{H}$ is nonunique since the influence of the unknown $\Sigma_{H}\left(\vec{r}_{0}\right)$ persists, can be obtained by selecting an upper and lower physically reasonable $\Sigma_{H}\left(\vec{r}_{0}\right)$ and integrating Eq. (17) with both along the respective characteristic.

Two situations can occur for which $\Sigma_{H}\left(\vec{r}_{0}\right)$ is known. First, there may be a direct pointwise conductance measurement available, or, second, an isolated point with $\vec{E}=0$ and $\nabla_{h} \cdot \vec{E} \neq 0$ [called "singular point", see Amm (1995)] is present at which $\Sigma_{H}=D / C$ is known from the input directly. From the singular points, characteristics emerge in all directions, and they are the only points where characteristics can meet (not intersect). In both cases, the points where $\Sigma_{H}\left(\vec{r}_{0}\right)$ is known will be selected as starting points for the integration along the characteristic(s) passing through it and make the solution of $\Sigma_{H}$ unique along them.

\subsection{Time-dependent situations}

It should be explicitly stated that the method of characteristics does not require $[\nabla \times \vec{E}]_{r}=0$. Thus, it is able to handle nonstationary situations when large time derivations of $\vec{B}$ cause a considerable amount of $[\nabla \times \vec{E}]_{r}$, as it may happen when large-magnitude plasma waves are incident to the ionosphere (e.g., Glaßmeier, 1988).

However, for the field separation and upward continuation in Sect. 2.2 we assumed that a magnetic potential exists, i.e., $\nabla \times \vec{B}=0$ holds. This neglects the displacement currents caused by $\partial \vec{E} / \partial t$ in nonstationary situations. For purity, one could treat the upward continuation in such a case as an inverse wave propagation problem and use methods similar to seismic migration to solve it. However, for several reasons we feel that this effort would be inadequate and unnecessary: (1) Between the ionosphere and the earth the waves travel as electromagnetic waves with phase and group velocity $c$, and need only $0.3 \mathrm{~ms}$ to reach the earth's surface. The typical sampling interval of magnetometers is $10 \mathrm{~s}$ (that of radars mostly longer). Thus, according to Nyquist's theorem the minimum cycle length of a wave resolvable is $20 \mathrm{~s}$. These waves can therefore complete only $1.5 \cdot 10^{-5}$ wave cycles. (2) On the spatial scale, magnetometer stations are typically not less than $100 \mathrm{~km}$ apart, so the minimum wavelength they can resolve is $200 \mathrm{~km}$. Thus, on a distance of $100 \mathrm{~km}$ between the ionosphere and the earth's surface, only half of a wavelength of the waves of interest can evolve. (1) and (2) together result in wave fronts that are quite exactly planar in the range of interest. (3) With approximate values of $\vec{E}=50 \mathrm{mV} / \mathrm{m}, \vec{B}=100 \mathrm{nT}$, $\partial \vec{E} / \partial t=10 \mathrm{mVm}^{-1} / 10 \mathrm{~s}$ and $\partial \vec{B} / \partial t=10 \mathrm{nT} / 10 \mathrm{~s}$, we get $\nabla \times \vec{B} /|\vec{B}| \approx 10^{-5} \nabla \times \vec{E} /|\vec{E}|$. Therefore neglecting $\nabla \times \vec{B}$ can be justified.

\section{Data analysis on a Harang-discontinuity situation}

In this section, we will apply the method of characteristics in spherical geometry as introduced in the previous section to a Harang-discontinuity (HD) situation on 27 October 1977. This event is particularly suitable to evaluate the results of our method since it was studied earlier by Lampen (1985) by means of a "trial and error" analysis. He composed observations between 16:50 and 18:10 UT to a single distribution using the "composite vector plot technique" [e.g., Untiedt and Baumjohann (1993), where the main results of Lampen's work are also shown]. To avoid uncertainties that are inherent to this technique even if most carefully done, i.e., the need of a stationarity assumption and the velocity estimation of the stationary structure, and to exploit the capabilities of our method, which does not rely on the reproduction of the ground magnetic field and thus does not need to estimate the ionospheric currents on a much larger area than that of actual interest, in this study we will perform a single time-step analysis. The need for such an analysis was also emphasized by Koskinen and Pulkkinen (1995) when they pointed out that the HD may move rapidly in latitude even within a few minutes. We will discuss some similarities and differences between the study of Lampen (1985) and ours further on in this section, and give a more general discussion of 
our results in the context of previous works on the HD in the next.

We use magnetic field data of the former Scandinavian Magnetometer Array (SMA) (Küppers et al., 1979) and electric field data of the STARE coherent-scatter radar (Nielsen, 1982). No all-sky camera data are available. The $\mathrm{Kp}$ index for the time under study is 5 (15-18 UT), followed by 7 for 18-24 UT. The magnetograms available suggest that our event takes place during the expansion phase of a substorm, and an average equatorward motion of the HD is expected. For our single time-step analysis, we choose the time 17:39 UT. At that time, the HD is located immediately above the densest area of the SMA, and STARE receives a good backscatter there. The latter fact is of special importance, since a single time-step analysis is mainly restricted to the immediate field of view of the measurement devices. In our case, the STARE field of view is much smaller than that of the SMA. Figure 3 shows the SMA ground magnetic, Fig. 4 the STARE electric field measurements. For the latter, we took only into account vectors with at least $6 \mathrm{~dB}$ of backscatter intensity for both STARE radars to exclude possible erratic vectors. The SMA data are shown in Fig. 3 as $90^{\circ}$ clockwise-rotated magnetic field vectors (cf. discussion in Sect. 2.2). An eastward electrojet can be seen over Scandinavia between about $62^{\circ}$ and $66^{\circ}$ latitude, with an increasing northward component towards north. Its maximum magnitude is about $250 \mathrm{nT}$ at $64^{\circ}$ latitude. Northward of that jet, the vectors turn to purely northern direction, indicative for the magnetic $\mathrm{HD}$, near $67.8^{\circ}$ latitude, with magnetic field disturbances near $70 \mathrm{nT}$. Further northward, a westward electrojet with a large northward deflection is observed with a slightly larger magnetic disturbance than at the HD reaching $100 \mathrm{nT}$.

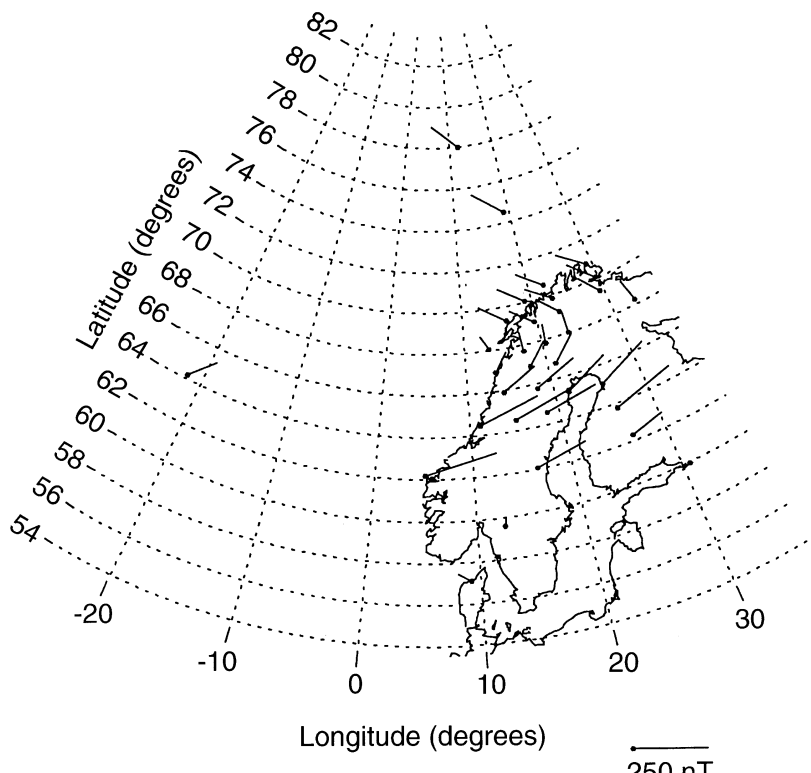

Fig. 3. By $90^{\circ}$ clockwise-related horizontal ground magnetic disturbance measurements of SMA and other ground magnetometers for 27 October 1979, 17:39 UT. Latitudes and longitudes are geographic
The STARE radar provides good backscatter between $67.6^{\circ}$ and about $71^{\circ}$ latitude (Fig. 4). The electric HD can be located quite precisely at $68.6^{\circ}$ latitude, where vectors are present which point nearly directly westward with about $25 \mathrm{mV} / \mathrm{m}$ of magnitude. The vectors south of the HD, pointing northward with slight westward deviation, have magnitudes of about $30 \mathrm{mV} /$ $\mathrm{m}$, whereas the vector population north of the HD between $69^{\circ}$ and $70^{\circ}$ latitude reaches up to $50 \mathrm{mV} / \mathrm{m}$ and points southward, with mostly small westward deviations, but also some eastward deflected vectors east of $19^{\circ}$ longitude.

We carry out our analysis in the area between $65^{\circ}$ and $71^{\circ}$ latitude and $15^{\circ}$ and $24^{\circ}$ longitude. This involves some southward extrapolation of the electric field in the eastward electrojet region, as was also applied by Lampen (1985). An inverse distance method was used for this extrapolation. This approach is justified since the electric field inside the eastward electrojet region has been reported as quite homogeneous (e.g., Baumjohann et al., 1980; Lühr et al., 1994). The final electric field distribution used as an input to our analysis is shown in Fig. 5b. Figure 5a shows the ionospheric equivalent currents $\vec{J}_{e q, I o n}$ as they result from the separation of the external part of the magnetic distrubance field, upward continuation of that part to the ionosphere and calculation of the equivalent currents as discussed in Sect. 2.2. In addition to the general increase in magnitude, a comparison with Fig. 3 indicates that the basic structure of the rotated ground magnetic vectors is preserved, but slight changes can be seen, e.g., in the northwest of the analysis area where the northern deflection of the westward pointing vectors is reduced. As a final input quantity, the modeled Hall-toPedersen conductance ratio $\alpha$ is displayed in Fig. 5c. A

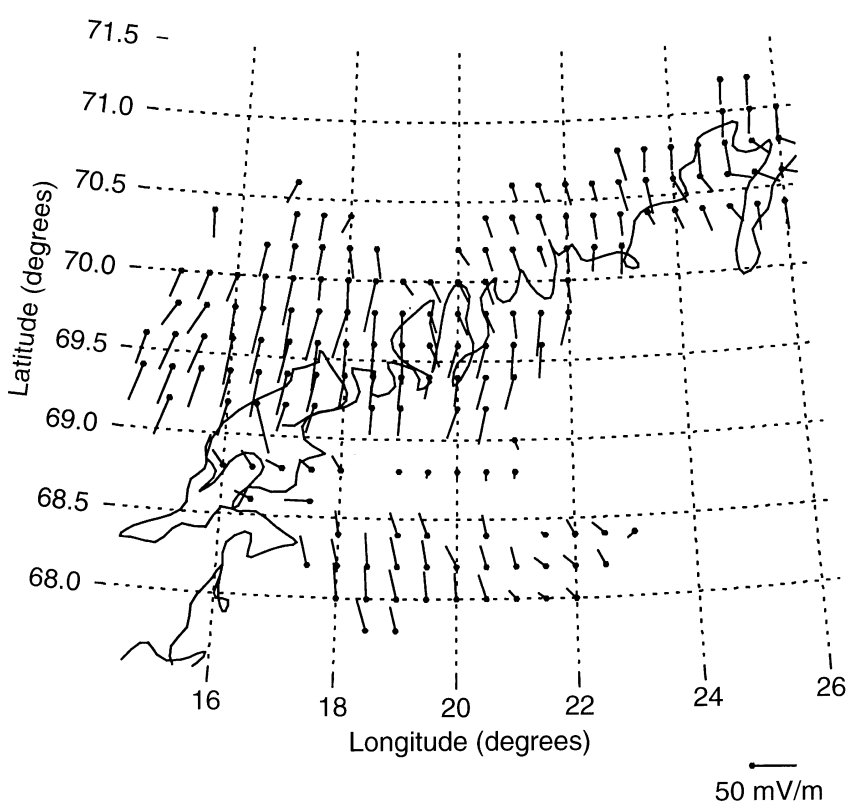

Fig. 4. STARE electric field data for 27 October 1979, 17:39 UT, with Scandinavian coastline (data courtesy of E. Nielsen, KatlenburgLindau) 


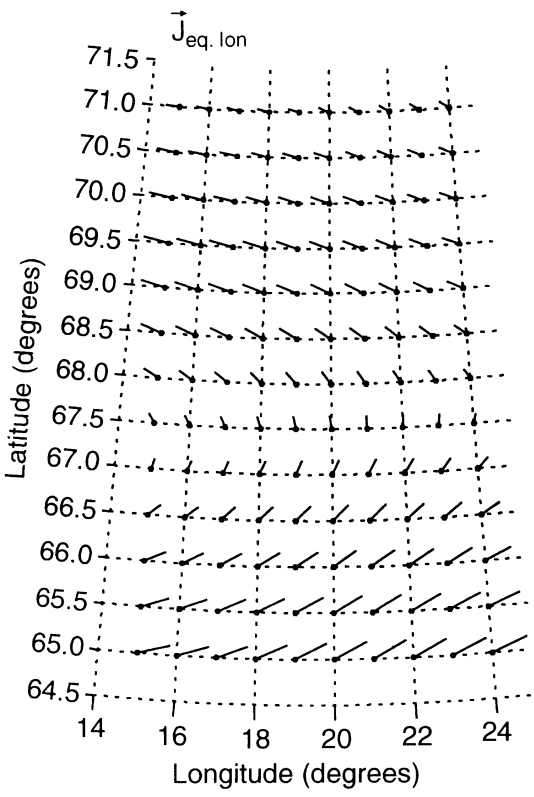

a $\quad 500 \overline{\mathrm{mA}} / \mathrm{m}$

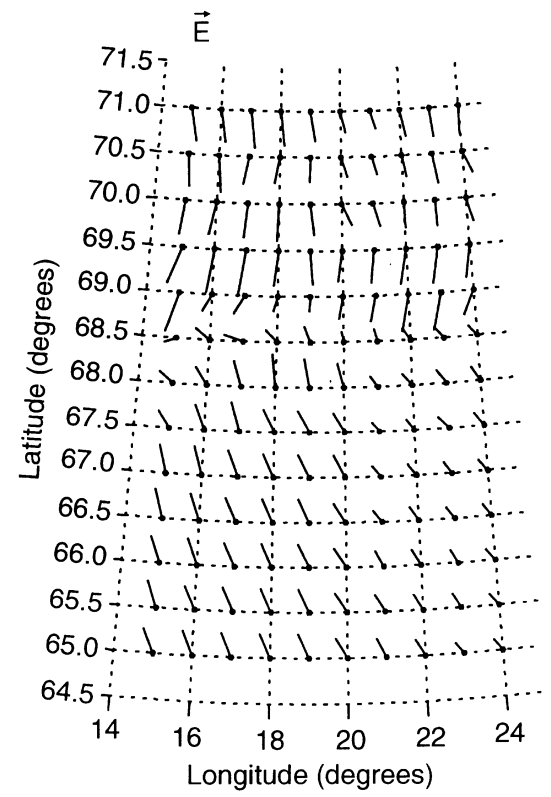

b $50 \overline{\mathrm{mV}} / \mathrm{m}$

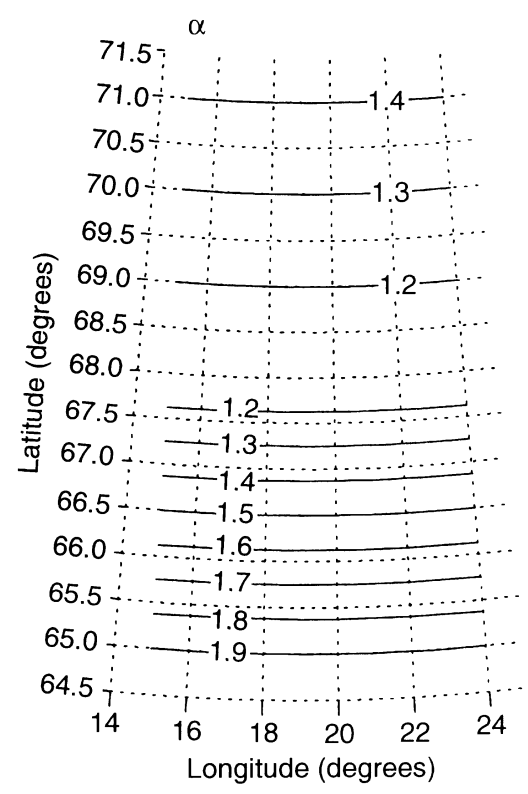

c
Fig. 5a-c. Input data for the method of characteristics. a Ionospheric equivalent currents, as resulted from separation of the external part of the magnetic disturbance field, its continuation to the ionospheric height, and transformation to equivalent currents. b Gridded electric

minimum of $\alpha$ of about 1.2 is modeled between the electric and magnetic HD at about $68.2^{\circ}$ latitude, with respect to the results of Lampen (1985) and Kunkel et al. (1986). From there, $\alpha$ increases to the south up to 2 in the eastward electrojet region. The smaller increase to the north was modeled with respect to the smaller increase in $\left|\vec{J}_{\text {eq,Ion }}\right|$ in that direction. No longitudinal variation is included. Note that this distribution is in fact the only modeled input to our analysis, and none of the later conclusions relies on this special distribution. It would be equally possible just to set a uniform median value of $\alpha$ (compare Amm, 1995), but we followed the previous studies, which both used a similar distribution with a minimum at and gradients perpendicular to the HD.

Two obvious differences to the study of Lampen (1985) are readily visible from the input distributions in Fig. 5a, (as well as from the data in Figs. 3 and 4): whereas in the earlier study the HD is northwestsoutheast aligned [as in the work of Kunkel et al. (1986) and also the statistical model of Heppner and Maynard (1987)], Figs. 4 and 5b show the HD to be almost aligned with geographical latitude, with even a slight northward tilt at the eastern side which results in an good alignment with geomagnetic latitude, too. Our observations agree with STARE data observations of Koskinen and Pulkkinen (1995, their Fig. 4), who found reasonable alignment of the HD with geomagnetic latitude for all six events that they studied. Next, in our single time-step observations the electric HD is found about $0.8^{\circ}\left( \pm 0.2^{\circ}\right)$ northward of the magnetic one as reported with similar values by Kamide and Vickrey (1983) and Kunkel et al. (1986), whereas in the composite vector plots of Lampen field. c Modeled distribution of Hall-to-Pedersen conductance ratio [modeled with respect to results of Lampen (1985) and Kunkel et al. (1986)]

(1985) the two almost coincide (cf. Untiedt and Baumjohann, 1993).

The output distributions of the method of characteristics are shown in Fig. 6. Figure 6a displays selected characteristics as defined in Eq. (16). As in previous studies in which this method was applied in planar geometry to an HD (Inhester et al., 1992; Amm, 1995), a strip of characteristics runs along the electric HD from which characteristics spread out to the north and south. Points of influencing boundary are marked as hatched squares. The influencing boundary takes up only about $25 \%$ of the whole boundary. The resulting $\Sigma_{H}$ is shown in Fig. 6b. For that figure, in regions where an uncertainty in $\Sigma_{H}$ persists due to the influence of unknown boundary values, we took the average of the upper and lower conductance estimate (see Sect. 2.4). Such regions are present in the southwest and in the outer northeast. The difference between the two estimates has a maximum value of about $5 \mathrm{~S}$ there. In Fig. 6b, a strong north-south gradient of $\Sigma_{H}$ is seen, with very low $\Sigma_{H}$ values mostly below $2 \mathrm{~S}$ in the region north of the electric HD. South of the magnetic $\mathrm{HD}, \Sigma_{H}$ increases considerably to reach values between 10 and $20 \mathrm{~S}$ at the southern boundary of our analysis region (i.e., still north of the center of the eastward jet, compare Fig. 3). The east-west gradient in $\Sigma_{H}$ in the eastward electrojet region is related to an opposite gradient in $|\vec{E}|$ (see Fig. 5b) and does not lead to a discontinuity in the resulting distribution of $\vec{J}$, as indicated by Fig. 6c. It is instructive to compare $\vec{J}$ with $\vec{J}_{e q, I o n}$ (Fig. 5a). The nearly symmetric distribution of $\vec{J}_{\text {eq,Ion }}$ with respect to the magnetic HD turns out to correspond to a highly assymetric distribution of $\vec{J}$ with large northeastward pointing current vectors up to 


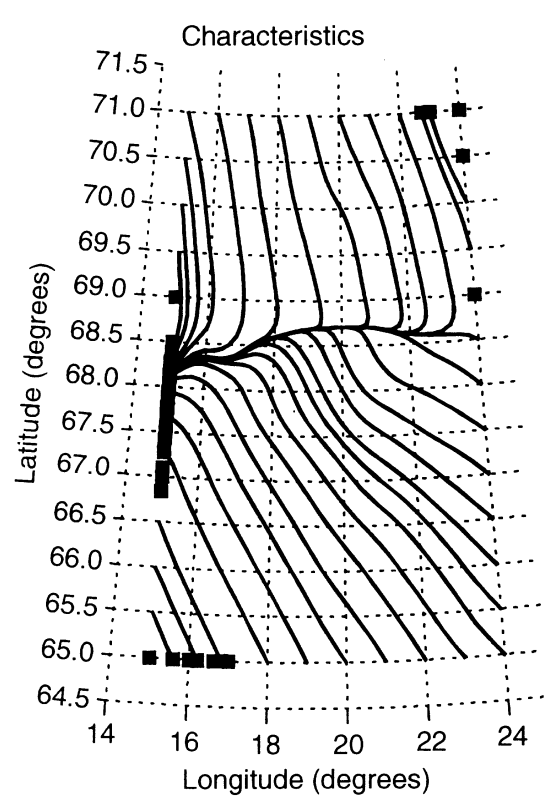

- Point of

influencing boundary

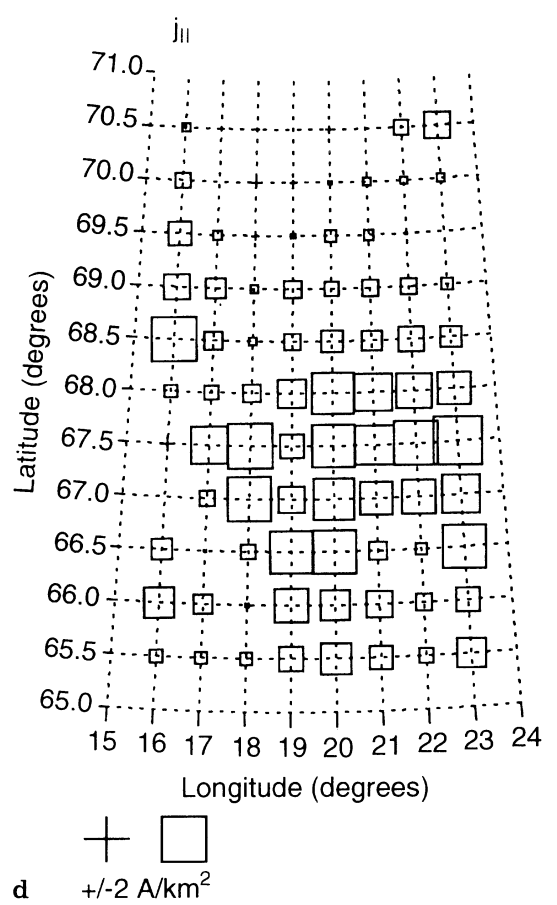

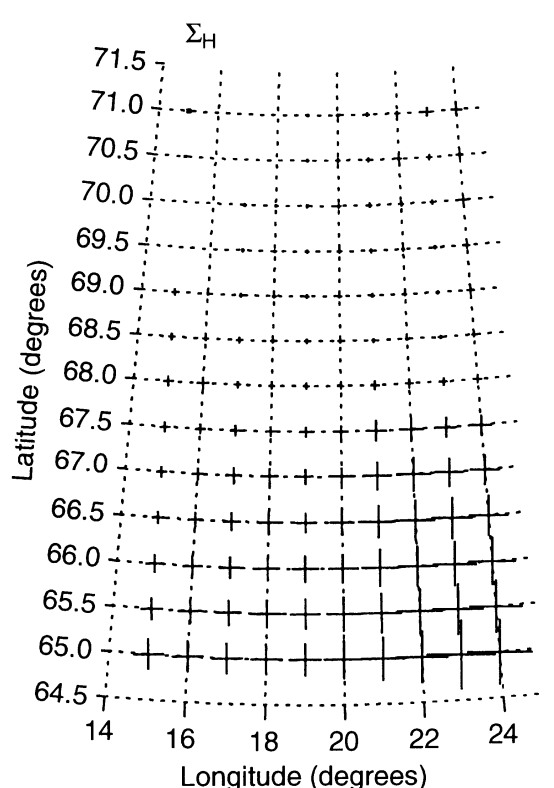

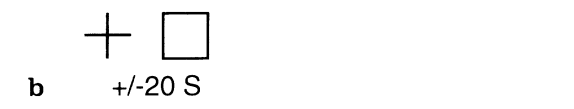

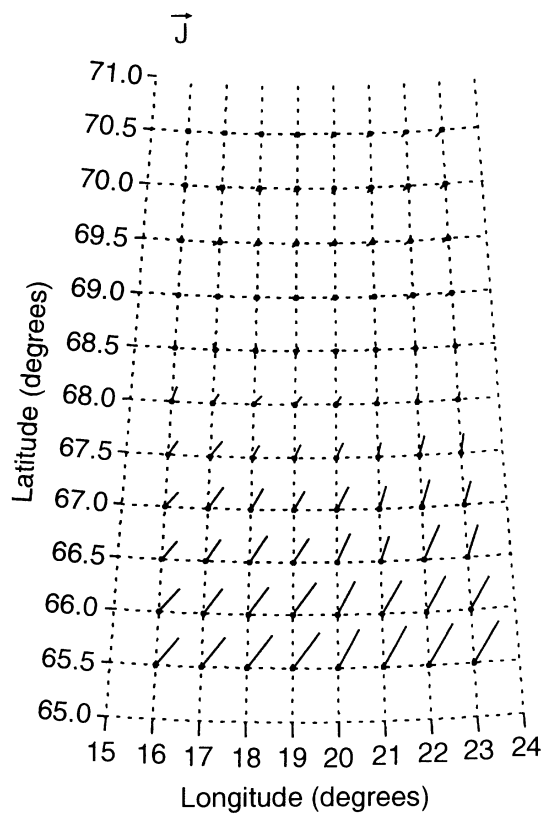

$500 \mathrm{~mA} / \mathrm{m}$

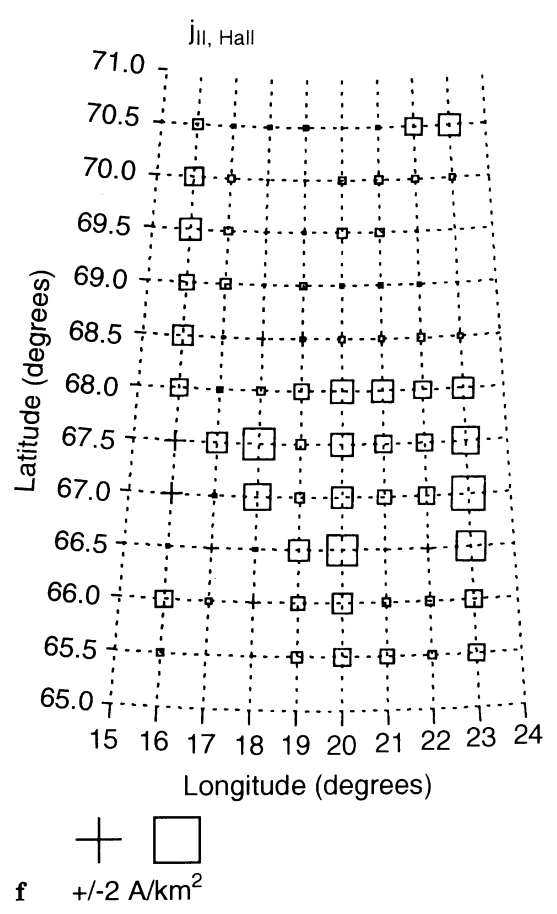

Fig. 6a-f. Results of the method of characteristics. a Characteristics; squares mark points of influencing boundary. b Hall conductance. c Ionospheric currents. d Total field- aligned currents. e Field-aligned

$450 \mathrm{~mA} / \mathrm{m}$ in the eastward electrojet region, but only very weak west-to-southwest-directed current vectors in the region north of the electric HD. Hence, the apparent westward electrojet in $\vec{J}_{e q, I o n}$ is caused by the effect of a sheet of large upward-flowing FACs up to $2 \mathrm{~A} / \mathrm{km}^{2}$ as shown in Fig. 6d. That sheet appears to have its center between the electric and the magnetic HD, but closer to the magnetic one. Upward FACs are visible in nearly the whole analysis area, but decrease strongly currents connected with Pedersen currents. f Field-aligned currents connected with Hall currents. Crosses mark downward, squares upward field-aligned currents

north of the electric HD. Slight downward FACs are also seen in between, but they are clearly weaker than the upward ones. The general picture of the FACs is quite patchy, but this is in fact what has been detected by satellite measurements above the HD, too (cf. Koskinen and Pulkkinen, 1995, and references therein). It would be interesting to compare our results with such measurements, but unfortunately none are available for this event. 
However, the patchy structure at least partly resolves when looking at the FACs which are connected to the Pedersen (Fig. 6e) and Hall (Fig. 6f) currents. Whereas the former are distributed relatively homogeneously south of the electric HD with magnitudes of $1 \mathrm{~A} / \mathrm{km}^{2}$ or less and disappear nearly completely north of it, the latter exhibit a more patchy behavior and have their highest intensities up to $1.5 \mathrm{~A} / \mathrm{km}^{2}$ near the latitude of the magnetic HD.

It is not surprising that our result distributions are not as even as those Lampen (1985) obtained with the "trial and error" method, or as any results of this method, because in "trial and error" one starts to model smooth and symmetric conductance distributions first and departs from them only if absolutely necessary. In contrast, we start out with plain data distributions except the one for $\alpha$ that has no decisive influence at all.

Still, in a broad view our results agree with that of Lampen (1985) in that a sheet of upward FACs is present along the HD and that the conductances are clearly larger in the eastward electrojet region than in the HD and north of it. On the other hand, in Lampen's study the strong decrease in $\Sigma_{H}$ is located considerably further south of the HD, which results in two distinctly separated sheets of upward FACs, one at the HD and one several degrees south of it, from which the latter is even the stronger. This feature is not supported by our results, nor by those of Kunkel et al., (1986), in another HD situation. In general, we feel that our output distributions show somewhat more detail than those provided by the "trial and error" method, and that the amount of assumptions and modeled input used is clearly reduced. Therefore, we feel encouraged to try to address some general questions on the HD in the next section.

\section{Discussion}

Only few previous single-event studies of the HD are available that involve two-dimensional analysis of all ionospheric electrodynamic parameters. The most extensive are those by Kunkel et al. (1986), later refined by Inhester et al. (1992) and Amm (1995), and that of Lampen (1985), shown also in Untiedt and Baumjohann (1993), on the same event as studied in the present paper. We will concentrate on these studies in our discussion. On the other hand, many works of different type (e.g., based on magnetometer or electric field measurements only, or using optical or satellite-based data, as well as statistical studies) have been carried out. These are summarized in the review of Koskinen and Pulkkinen (1995). We will use the questions raised in that paper as a starting point of our discussion.

One question already mentioned in the previous section is whether the HD is tilted against latitude in northwest-southeast direction as indicated by the composite vector plot results of Lampen (1985) and Kunkel et al. (1986), as well as by the statistical results of Heppner and Maynard (1987), or if it is mainly aligned with geomagnetic latitudes, as it was proposed by Koskinen and Pulkkinen (1995) who studied STARE single time-step data of a number of HD events. Our data set clearly supports the latter point of view. We have already discussed the possible shortcomings of the composite vector plot technique. Marklund (1993) pointed out that statistical pictures may be quite different from single-event results.

Another question is whether or not there is plasma flow across the HD. The STARE data of our event (Fig. 4) shows some nearly purely westward directed electric field vectors in the center of the electric HD, clearly indicating a southward plasma flow across the HD.

However, in the context of currents, parts c and d of Fig. 6 indicate that, at least in the situation studied here, almost all of the eastward electrojet current, both regarding the Hall and Pedersen parts is diverted into FACs at or south of the electric HD. This agrees with the conclusions of Lampen (1985) and Kunkel et al. (1986), but is in contrast to the proposal raised by Kamide (1978) of current continuity without FACs at the HD.

When comparing our results to the sketch of the large-scale electrodynamics of the HD presented by Koskinen and Pulkkinen (1995; their Fig. 7), a general agreement with our results can be stated, except for two points: (1) The FACs connected with the Pedersen currents are shown in their sketch as being restricted to the immediate environment of the HD and spatially separated from the FACs connected with the Hall currents. Our results indicate that due to the conductance gradient perpendicular to the HD, the FACs connected with the Pedersen currents are fairly evenly distributed over a broad area south of the electric HD, overlapping with the location of the maximum Hall current FACs. The latter are located south of the electric HD (regarding the Hall currents of the eastward electrojet), in agreement with the picture of Koskinen and Pulkkinen (1995). (2) Since the westward electrojet is very weak in the situation we studied, its contribution to FACs is negligible. This is in contrast to the symmetric picture of Koskinen and Pulkkinen (1995) regarding the position and strength of the jets and its FACs to both sides of the HD. However, we admit that these results may be a feature of the special situation under study, and it is not clear whether they can be generalized. It may well be that a stronger westward electrojet exists north of our analysis region, in accordance with the results of Lampen (1985).

Another question raised by Koskinen and Pulkkinen (1995) is why the break-up arcs are often closer to the magnetically defined HD than to the electrically defined one. Although a complete answer to this question is not possible from ionospheric results only, it is interesting to note that the FACs connected to the Hall currents (Fig. 6f) peak exactly in the region between the electrically and the magnetically defined HD, closer to the latter. Since the substorm current wedge is believed to be mainly constituted by Hall currents (e.g., Kamide and Baumjohann, 1993), it seems reasonable that the breakup starts where they are fed from the magnetosphere.

Finally, another question is why the magnetic HD (as defined by ground magnetometers) is typically located 
$1-2^{\circ}$ south of the electric one. By recalculating the ground magnetic field from our results by Biot-Savart integration, we found that if the effect of the horizontal ionospheric currents only is included, the magnetic HD would shift even north of the electric one, due to the much larger strength of the eastward electrojet compared to the westward one in our event. The picture changes when we include the magnetic effect of the FACs: although the calculation of $\vec{B}_{G}$ from the currents inside our analysis region only cannot completely regenerate the true $\vec{B}_{G}$, since the effect of the currents outside that region is missing, it became clear that the main effect in the southward shift of the magnetic HD comes from the east-west-aligned sheet of FACs connected to the Hall currents. This effect is most intense if there is a positive west-east gradient in absolute value of the magnitude of these FACs, as seen in Fig. $6 f$.

We would like to stress that we do not claim our results obtained from a single snapshot analysis to be necessarily general for the whole HD event on 27 October 1977, or for all HD events. However, concerning the orientation of the HD, all available snapshots of the electric field for our event show the same good alignment with geomagnetic latitude as described in the preceding.

From the data available for this event, it is difficult to build up a time-series of similar analyses as presented in Sect. 3, as this would require the study of the dynamic evolution of the HD. For later times than studied here, the HD soon moves out of the STARE field of view to the south, and for earlier times it is soon located north of the SMA network. Moreover, the amount of backscatter received by STARE varies. Such a time-series analysis could thus only be performed if electric field data would be available on approximately the same area as the magnetometer data. At the moment, efforts are made to use SuperDARN data in such a way, and results will be published in the near future. In that kind of analysis, the advantage of the use of spherical geometry will become more obvious, too. The event studied in this paper could still be handled in planar geometry, but it was chosen in order to compare our method with the earlier "trial and error" method, and because of the interesting physical situation. Moreover, with our study we have shown that the method of characteristics in spherical geometry can be regarded as a superset of the planar version in the sense that it can also be applied to smaller areas as earlier typically analyzed in planar geometry.

Future work will include the application of our method to different types of ionospheric electrodynamic situations and to larger regions of study.

\section{Summary}

Regarding the methodical aspects of this paper, we can summarize our results as follows:

1. The method of characteristics is available in spherical geometry for use on large analysis areas in the auroral zone, including tools for magnetic field separation, upward continuation, and calculation of ionospheric equivalent currents. The spherical version of the method can be used for smaller regions as previously studied by planar geometry methods as well.

2. The amount of assumptions and modeled input is clearly reduced as compared to the earlier "trial and error" method, and the output distributions reveal more details, thus allowing a more detailed physical interpretation. Since the method of characteristics is a forward method, it is also much faster than any fitting method. However, since divergence and curl of input quantities (or quantities derived from them) have to be estimated, good two-dimensional input data coverage of $\vec{E}$ and $\vec{B}_{G}$ is required for instantaneous time-step analyses.

The main results of the application of our method to a Harang discontinuity situation on 27 October 1977, 17:39, UT are:

1. In general, our study supports the results of earlier ones in that the conductances in the HD region and north of it are clearly reduced compared to the eastward electrojet region south of it, and that most of the current is diverted into FACs at the HD, although plasma flow is observed through it.

2. The FACs connected with the Pedersen currents show a nearly uniform distribution south of the electric HD with magnitudes around $1 \mathrm{~A} / \mathrm{km}^{2}$. In contrast, the FACs connected with the Hall currents peak in the region between the electric and magnetic $\mathrm{HD}$, closer to the magnetic one, with magnitudes up to $1.5 \mathrm{~A} / \mathrm{km}^{2}$, in the same area where initial break-ups are often observed.

3. The FACs connected with the Hall currents form a latitudinally aligned sheet and are mainly responsible for the southward shift of the magnetically defined HD with respect to the electrically defined one by $1-2^{\circ}$ of latitude. That shift is enhanced if a west-east gradient in absolute value of the magnitude of those FACs is present.

4. The HD shows no noticeable tilt against geomagnetic latitude.

Acknowledgements. The author likes to thank K.H. Glaßmeier (Braunschweig) for his steady encouragement and help during this work. He is also grateful to B. Inhester (Katlenburg-Lindau), H. Koskinen and A. Viljanen (both Helsinki), and J. Untiedt (Münster) for valuable discussions or comments. The STARE data used in this paper have kindly be provided by E. Nielsen (Katlenburg-Lindau) whose support is gratefully acknowledged. This work was supported by a DAAD fellowship HSP II financed by the German Federal Ministry for Research and Technology and a grant from the German Science Foundation.

Topical Editor D. Alcaydé thanks V.O. Papitashvili and M. Lester for their help in evaluating this paper.

\section{References}

Aikio, A. T., and K. U. Kaila, A substorm observed by EISCAT and other ground-based instruments - evidence for near-Earth substorm initiation, J. Atmos. Terr. Phys, 58, 5, 1996. 
Amm, O., Direct determination of the local ionospheric Hall conductance distribution from two-dimensional electric and magnetic field data: application of the method using models of typical ionospheric electrodynamic situations, J. Geophys. Res., 100, 24173, 1995.

Amm, O., Comment on "A three-dimensional, iterative mapping procedure for the implementation of an ionosphere-magnetosphere anisotropic Ohm's law boundary condition in global magnetohydrodynamic simulations" by Michael L. Goodman, Ann. Geophysicae, 14, 773, 1996.

Amm, O., Ionospheric elementary current systems in spherical coordinates and their application. J. Geomagn. Geoelectr., 49, 947, 1997.

Baumjohann, W., J. Untiedt, and R. A. Greenwald, Joint twodimensional observations of ground magnetic and ionospheric electric fields associated with auroral zone currents: 1. threedimensional current flows associated with a substorm-intensified eastward electrojet, J. Geophys. Res., 85, 1963, 1980.

Baumjohann, W., R. J. Pellinen, H. J. Opgenoorth, and E. Nielsen, Joint two-dimensional observations of ground magnetic and ionospheric electric fields associated with auroral zone currents: current system associated with local auroral break-ups, Planet. Space Sci., 29, 431, 1981.

Chapman, S., and J. Bartels, Geomagnetism, vol. II, Oxford University Press, New York, 1940.

Fukushima, N., Generalized theorem of no ground magnetic effect of vertical currents connected with Pedersen currents in the uniform conducting ionosphere, Rep. Ionos. Space Res. Jpn., 60, 35, 1976.

Glassmeier, K. H., Reconstruction of the ionospheric influence on ground-based observations of a short-duration ULF pulsation event, Planet. Space Sci., 36, 801, 1988.

Greenwald, R. A., K. B. Baker, J. R. Dudeney, M. Pinnock, T. B. Jones, E. C. Thomas, J.-P. Villain, J.-C. Cerisier, C. Senior, C. Hanuise, R. D. Hunsucker, G. Sofko, J. Koehler, E. Nielsen, R. Pellinen, A. D. M. Walker, N. Sato, and H. Yamagishi, DARN/ SuperDARN: a global view of the dynamics of high-latitude convection, Space Sci. Rev., 71, 761, 1995.

Haines, G. V., Spherical cap harmonic analysis, J. Geophys. Res., 90, 2583, 1985 .

Haines, G. V., Computer programs for spherical cap harmonic analysis of potential and general fields, Comp. Geosci., 14, 413, 1988.

Haines, G. V., and J. M. Torta, Determination of equivalent current sources from spherical cap harmonic models of geomagnetic field variations, Geophys. J. Int., 118, 499, 1994.

Heppner, J. P., and N. C. Maynard, Empirical high-latitude electric field models, J. Geophys. Res., 92, 4467, 1987.

Hobson, E. W., The theory of spherical and ellipsoidal harmonics, Cambridge University Press, New York, 1931.

Inhester, B., J. Untiedt, M. Segatz, and M. Kürschner, Direct determination of the local ionospheric Hall conductance distribution from two-dimensional electric and magnetic field data, J. Geophys. Res., 97, 4073, 1992.

Kamide, Y., On current continuity at the Harang discontinuity, Planet. Space Sci., 26, 237, 1978.

Kamide, Y., and W. Baumjohann, Magnetosphere-ionosphere coupling, Springer, Berlin, Heidelberg, New York, 1993.

Kamide, Y., and J. F. Vickrey, Variability of the Harang discontinuity as observed by the Chatanika radar and the IMS Alaska magnetometer chain, Geophys. Res. Lett., 10, 159, 1983.

Kamide, Y., M. Kanamitsu, and S.-I. Akasofu, A new method of mapping worldwide potential contours for ground magnetic perturbations: equivalent current representation, J. Geophys. Res., 81, 3810, 1976.

Kamide, Y., A. D. Richmond, and S. Matsushita, Estimation of ionospheric electric fields, ionospheric currents and field-aligned currents from ground magnetic records, J. Geophys. Res., 86, $801,1981$.
Kirkwood, S., H. J. Opgenoorth, and J. S. Murphree, Ionospheric conductivities, electric fields and currents associated with auroral substorms measured by the EISCAT radar, Planet. Space Sci., 36, 1359, 1988.

Koskinen, H. E. J., and T. I. Pulkkinen, Midnight velocity shear zone and the concept of Harang discontinuity, J. Geophys. Res., 100, 9539, 1995.

Kunkel, T., W. Baumjohann, U. Untiedt, and R. A. Greenwald, Electric fields and currents at the Harang discontinuity: a casestudy, J. Geophys., 59, 73, 1986.

Küppers, F., J. Untiedt, W. Baumjohann, K. Lange, and A. G. Jones, A two-dimensional magnetometer array for ground-based observations of auroral zone electric currents during the International Magnetospheric Study (IMS), J. Geophys., 46, 429, 1979.

Lampen, M., Elektrodynamische Parameter in der Umgebung der Harang-Diskontinuität, untersucht anhand kombinierter Messungen der magnetischen Störungen am Boden und ionsophärischer elektrischer Felder im frühen Abendsektor des 27. Oktober 1977, Diploma thesis, University of Münster, Münster, Germany, 1985.

Lester, M., J. A. Davies, and T. S. Virdi, High-latitude Hall and Pedersen conductances during substorm activity in the SUNDIAL-ATLAS campaign, J. Geophys. Res., 101, 26719, 1996.

Lühr, H. and K. Schlegel, Combined measurements of EISCAT and the EISCAT magnetometer cross to study $\Omega$ bands, J. Geophys. Res., 99, 8921, 1994.

Lühr, H., H. Geisler, and K. Schlegel, Current density models of the eastward electrojet derived from ground-based magnetic field and radar measurements, J. Atmos. Terr. Phys., 56, 81, 1994.

Marklund, G., Viking investigations of auroral electrodynamical processes, J. Geophys. Res., 98, 1691, 1993.

Nielsen, E., The STARE system and some of its applications, in The IMS source book: guide to the international magnetosphere study data analysis, Ed. C. T. Russell and D. J. Southwood, AGU, Washington DC, pp. 213-224 1982.

Olsson, A., M. A. L. Persson, H. J. Opgenoorth, and S. Kirkwood, Particle precipitation in auroral breakups and westward traveling surges, J. Geophys. Res., 101, 24661, 1996.

Opgenoorth, H. J., R. J. Pellinen, W. Baumjohann, E. Nielsen, G. Marklund, and L. Eliasson, Three-dimensional current flow and particle precipitation in a westward traveling surge (observed during the Barium-GEOS rocket experiment), J. Geophys. Res., 88, 3138, 1983.

Papitashvili, V. O., B. A. Belov, D. S. Faermark, Ya. I. Feldstein, S. A. Golyshev, L. I. Gromova, and A. E. Levitin, Electric potential patterns in the northern and southern polar regions parametrized by the interplanetary magnetic field, J. Geophys. Res., 99, $13251,1994$.

Richmond, A. D., The computation of magnetic effects of fieldaligned magnetospheric currents, J. Atmos. Terr. Phys., 36, 245, 1974.

Richmond, A. D., and Y. Kamide, Mapping electrodynamic features of the high-latitude ionosphere from localized observations: technique, J. Geophys. Res., 93, 5741, 1988.

Schlegel, K., Auroral zone E-region conductivities during solar minimum derived from EISCAT data, Ann Geophysicae, 6, 129, 1988.

Stauning, P., Progressing IMF $\mathrm{B}_{\mathrm{y}}$-related polar ionospheric convection disturbances, J. Geomagn. Geoelectr., 47, 735, 1995.

Tamao, T., Direct contribution of oblique field-aligned currents to ground magnetic fields, J. Geophys. Res., 91, 183, 1986.

Torta, J. M., and A. de Santis, On the derivation of the Earth's conductivity structure by means of a spherical cap harmonic analysis, Geophys. J. Int, 127, 441, 1996.

Untiedt, J., and W. Baumjohann, Studies of polar current systems using the IMS Scandinavian magnetometer array, Space Sci. Rev., 63, 245, 1993. 